\title{
Effect of Pnictogen Height on Spin Waves in Iron Pnictides
}

\author{
Chenglin Zhang, ${ }^{1,2}$ Leland W. Harriger, ${ }^{3}$ Zhiping Yin, ${ }^{4}$ Weicheng Lv, ${ }^{2}$ Miaoyin Wang, ${ }^{2}$ Guotai Tan, ${ }^{2}$ Yu Song, ${ }^{1}$ \\ D. L. Abernathy, ${ }^{5}$ Wei Tian, ${ }^{5}$ Takeshi Egami ${ }^{2,5,6}$ Kristjan Haule, ${ }^{4}$ Gabriel Kotliar, ${ }^{4}$ and Pengcheng Dai ${ }^{1, *}$ \\ ${ }^{1}$ Department of Physics and Astronomy, Rice University, Houston, Texas 77005, USA \\ ${ }^{2}$ Department of Physics and Astronomy, The University of Tennessee, Knoxville, Tennessee 37996-1200, USA \\ ${ }^{3}$ NIST Center for Neutron Research, National Institute of Standards and Technology, Gaithersburg, Maryland 20899, USA \\ ${ }^{4}$ Department of Physics, Rutgers University, Piscataway, New Jersey 08854, USA \\ ${ }^{5}$ Quantum Condensed Matter Division, Oak Ridge National Laboratory, Oak Ridge, Tennessee 37831, USA \\ ${ }^{6}$ Department of Materials Science and Engineering, The University of Tennessee, Knoxville, Tennessee 37996-1200, USA
}

(Received 2 April 2014; published 28 May 2014)

\begin{abstract}
We use inelastic neutron scattering to study spin waves in the antiferromagnetic ordered phase of iron pnictide $\mathrm{NaFe}$ ss throughout the Brillouin zone. Comparing with the well-studied $A \mathrm{Fe}_{2} \mathrm{As}_{2}(A=\mathrm{Ca}, \mathrm{Sr}$, $\mathrm{Ba}$ ) family, spin waves in $\mathrm{NaFeAs}$ have considerably lower zone boundary energies and more isotropic effective in-plane magnetic exchange couplings. These results are consistent with calculations from a combined density functional theory and dynamical mean field theory and provide strong evidence that pnictogen height controls the strength of electron-electron correlations and consequently the effective bandwidth of magnetic excitations.
\end{abstract}

DOI: 10.1103/PhysRevLett.112.217202

PACS numbers: 75.30.Ds, 75.50.Ee

Shortly after the discovery of the collinear antiferromagnetic (AF) order in the parent compounds of iron pnictide superconductors [Fig. 1(a)] [1-6], a key issue was raised concerning the strength of electron correlations in these materials and their relationship to magnetism and superconductivity [7-13]. In the itinerant picture, the static AF order arises from the nesting of the hole and electron Fermi surfaces $[7,8]$, much like the spin-density-wave state of chromium [14]. Here, superconductivity occurs due to the sign-reversed superconducting order parameter between the hole and electron Fermi surfaces [15,16]. Alternatively, iron pnictides may be in close proximity to a Mott-insulating phase [10-12], and therefore local physics and electron correlations are important to the magnetism and superconductivity. In this picture, the local moments formed by the Fe $3 d$ electrons, especially those in the $d_{x z}, d_{y z}$, and $d_{x y}$ orbitals, are coupled to their neighbors by anisotropic superexchange interactions, which are also responsible for the emergence of superconductivity [Figs. 1(c)-1(f)] [17-21].

Although there are indeed electron correlations in iron pnictides [22,23], the correlation strength arises primarily from the Hund's coupling $J_{H}$, which tends to align spins of all the electrons on a given Fe-atom, and hence enhances spin fluctuations without considerably impeding charge excitations [24,25]. This is in contrast to the Coulomb repulsion $U$, which severely hampers charge excitations in order to enhance spin fluctuations [26]. Due to the slow spin fluctuations in iron pnictides, the local magnetic moments appear on $\mathrm{Fe}$ sites, but due to fast charge fluctuations, these local moments are considerably reduced from the atomic-limit value, and only a fraction of the local moments order statically in the AF state $[27,28]$. Therefore, electronic excitations at low energy are neither fully itinerant nor fully localized, but have a dual nature, and are showing characteristic of both [6]. Density functional theory (DFT) combined with dynamical mean field theory (DMFT) may provide a realistic approach to describe the partially itinerant and partially localized electrons in iron pnictides [29,30]. With this approach, the evolutions of spin excitations in electron and hole-doped $\mathrm{BaFe}_{2} \mathrm{As}_{2}$ has been mapped out $[28,31,32]$. Furthermore, the method predicted that the correlation strength depends sensitively on the Fepnictogen distance owing to the hopping mechanism of the Fe $3 d$ electrons, and is strongly enhanced with increasing Fe-pnictogen height $[25,33]$. Experimentally, spin waves throughout the Brillouin zone have only been measured in the $A \mathrm{Fe}_{2} \mathrm{As}_{2}(A=\mathrm{Ba}, \mathrm{Sr}, \mathrm{Ca})$ family due to the availability of large single crystals [34-38]. Since the pnictogen heights [5] and spin-wave bandwidths [6] are similar for $A \mathrm{Fe}_{2} \mathrm{As}_{2}$, it remained unclear whether the iron-pnictogen distance in iron pnictides can indeed control the electron correlations and spin excitation spectra.

In this Letter, we present inelastic neutron scattering (INS) studies of spin waves in NaFeAs, the parent compound of $\mathrm{NaFe}_{1-x} \mathrm{Co}_{x}$ As family of iron pnictide superconductors [39]. We chose $\mathrm{NaFeAs}$ because it has weak AF ordered moment $\left(\mu \approx 0.1 \mu_{B}\right)$, low Néel temperature $\left[T_{N}=45 \mathrm{~K}\right.$, Fig. 1(b)], and large pnictogen height $\left(h_{M A s}=1.416 \AA\right)$ [4], significantly different from previously studied $A \mathrm{Fe}_{2} \mathrm{As}_{2}\left(\mu \approx 1 \mu_{B}, \quad T_{N} \geq 138 \mathrm{~K}\right.$, and $h_{M A s} \approx 1.358 \AA$ ) [Fig. 1(c)] [5]. Although the in-plane Fe-Fe distance may also play a role in determining the electronic properties of the system, its change from 

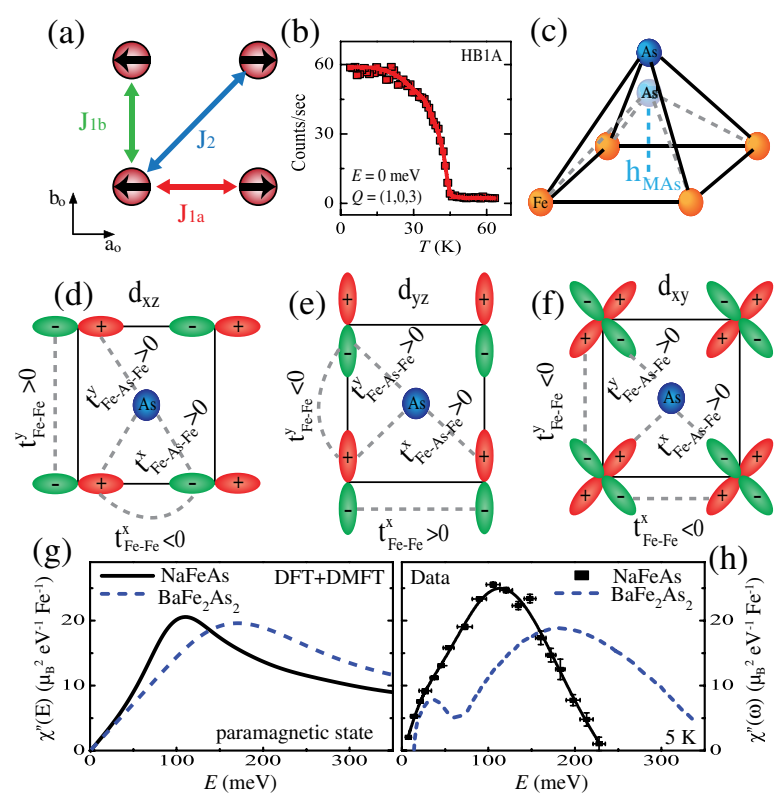

FIG. 1 (color online). (a) Schematic diagram of the Fe spin ordering in $\mathrm{NaFeAs}$ with the effective magnetic nearest-neighbor, next-nearest-neighbor exchange couplings, $J_{1 a}, J_{1 b}$ and $J_{2} \cdot a_{o}$ and $b_{o}$ mark the orthorhombic lattice structure of the system. (b) Temperature dependence of the AF Bragg peak intensity showing $T_{N}=45 \mathrm{~K}$. (c) Schematic diagram of the FeAs tetrahedron, showing increased iron pnictogen height $\left(h_{M A s}\right)$ from $\mathrm{BaFe}_{2} \mathrm{As}_{2}$ to NaFeAs $(\sim 0.058 \AA)$. The in-plane Fe-Fe distance changes are much smaller $\left(\sim 0.011 \AA\right.$ ) from $\mathrm{BaFe}_{2} \mathrm{As}_{2}(2.802 \AA)$ to $\mathrm{NaFeAs}(2.791 \AA)$. Schematic diagrams illustrating the direct and indirect electron hoppings of (d) $d_{x z}$, (e) $d_{y z}$, and (f) $d_{x y}$ orbitals. (g) Calculated energy dependence of the local dynamic spin susceptibility $\chi^{\prime \prime}(E)$ per Fe for $\mathrm{BaFe}_{2} \mathrm{As}_{2}$ and $\mathrm{NaFeAs}$ as obtained from the combined DFT and DMFT method [31]. (h) Energy dependence of the measured local dynamic spin susceptibility for $\mathrm{NaFeAs}$, and $\mathrm{BaFe}_{2} \mathrm{As}_{2}$ [46].

$A \mathrm{Fe}_{2} \mathrm{As}_{2}$ to $\mathrm{NaFeAs}$ is much smaller. If electron correlations and spin-wave spectra in iron pnictides are mostly controlled by the pnictogen heights in these materials, we expect stronger electron correlations and narrower spin-wave bandwidth in $\mathrm{NaFeAs}$ compared with that of $A \mathrm{Fe}_{2} \mathrm{As}_{2}$. Indeed, we find that spin waves in $\mathrm{NaFeAs}$ have the zone boundary energy near $\sim 100 \mathrm{meV}$, much lower than the $\sim 200 \mathrm{meV}$ zone boundary energy of $A \mathrm{Fe}_{2} \mathrm{As}_{2}$ [35-37]. By fitting the spin wave spectra using a similar Heisenberg Hamiltonian model as to $A \mathrm{Fe}_{2} \mathrm{As}_{2}[35,36]$, we find that the nearest-neighbor magnetic exchange couplings in $\mathrm{NaFeAs}$ are also more isotropic. These results are consistent with DFT + DMFT calculations, suggesting that the increased pnictogen height in $\mathrm{NaFeAs}$ increases the electron correlations, especially in the $d_{x y}$ orbitals, and can fundamentally affect the spin dynamical properties in these materials.

Single crystals of NaFeAs were grown by a self-flux method as described previously [40,41]. Neutron diffraction measurements on the crystals reveal an AF transition at
$T_{N}=45 \mathrm{~K}$ [Fig. 1(b)] [42]. Our INS experiments were carried out on the ARCS chopper spectrometer at the Spallation Neutron Source (SNS), Oak Ridge National Laboratory. We coaligned $\sim 17 \mathrm{~g}$ of single crystals in the $[H H L]$ scattering plane with a mosaic $<3^{\circ}$. The wave vector $\mathbf{q}$ at $\left(q_{x}, q_{y}, q_{z}\right)$ in $\AA^{-1}$ is defined as $(H, K, L)=$ $\left(q_{x} a_{o} / 2 \pi, q_{y} b_{o} / 2 \pi, q_{z} c / 2 \pi\right)$ where $a_{o} \approx b_{o} \approx 5.5664 \AA$ and $c=2 \times 6.96 \AA$ using the orthorhombic magnetic unit cell. In this notation, the AF Bragg peaks occur at the $(1,0, L)$ positions with $L=1,3, \ldots$ [4]. For our measurements, the incident beam energies were $E_{i}=80,150$, $250 \mathrm{meV}$ with $k_{i}$ parallel to the $c$-axis. Spin-wave intensity was normalized to absolute units using a vanadium standard ( $50 \%$ error).

For comparison with our INS experiments, we calculate the energy and wave vector dependence of the imaginary part of the dynamic spin susceptibility $\chi^{\prime \prime}(\mathbf{q}, E)$ [43] using a DFT + DMFT method, where $E=\hbar \omega$ [28,32,33]. Figure $1(\mathrm{~g})$ shows the energy dependence of the local dynamic spin susceptibility $\chi^{\prime \prime}(E)$ per $\mathrm{Fe}$ for NaFeAs and $\mathrm{BaFe}_{2} \mathrm{As}_{2}$ where $\chi^{\prime \prime}(E)$ per formula unit is defined as $\chi^{\prime \prime}(E)=\int \chi^{\prime \prime}(\mathbf{q}, E) d \mathbf{q} / \int d \mathbf{q}[28,32]$. We see that the effect of increasing the pnictogen height from $A \mathrm{Fe}_{2} \mathrm{As}_{2}$ to $\mathrm{NaFeAs}$ is expected to suppress high-energy spin waves and shift the spectral weight to lower energies, thus reducing the spin-wave bandwidth and increasing electron correlations $[25,31,33]$. The outcome of the experiments suggests that the spin-wave bandwidth of NaFeAs is reduced nearly by half from that of $\mathrm{BaFe}_{2} \mathrm{As}_{2}$ [Fig. 1(h)].

To substantiate the experimental and theoretical results presented in Fig. 1, we show in Fig. 2 the two dimensional constant-energy $(E)$ images of spin waves of $\mathrm{NaFeAs}$ in the $(H, K)$ scattering plane at different energies and their comparison with fits from Heisenberg Hamiltonian and DFT + DMFT calculations. In the AF ordered state, spin waves in NaFeAs have an anisotropy spin gap of $\Delta=$ $3 \mathrm{meV}$ at the AF zone center $[38,42]$. Figures 2(a)-2(e) show the evolution of spin waves at energy transfers of $E=15 \pm 3,45 \pm 3,60 \pm 10,80 \pm 10$, and $100 \pm 10 \mathrm{meV}$, respectively. At $E=15 \pm 3 \mathrm{meV}$ [Fig. 2(a)], spin waves form transversely elongated ellipses centered at the in-plane $\mathrm{AF}$ zone centers $( \pm 1,0)$ and $(0, \pm 1)$ for the twinned domains of NaFeAs. On increasing the energies to $E=$ $45 \pm 3$ and $60 \pm 10 \mathrm{meV}$, spin waves split transversely [Figs. 2(b) and 2(c)] from the AF zone centers with additional excitations appearing near the $( \pm 1, \pm 1)$ positions. On further increasing the energy to $E=80 \pm 10 \mathrm{meV}$, spin waves become crosslike excitations around $( \pm 1, \pm 1)$ [Fig. 2(d)]. Finally, spin waves at $E=100 \pm 10 \mathrm{meV}$ [Fig. 2(e)] near the zone boundary form anisotropic and asymmetric rings around $( \pm 1, \pm 1)$ similar to the zone boundary spin waves of $\mathrm{BaFe}_{2} \mathrm{As}_{2}$ at $E=157 \pm 10 \mathrm{meV}$ [36].

We have attempted but failed to fit the entire spin-wave spectra in Figs. 2(a)-2(e) using a Heisenberg Hamiltonian consisting of the effective in-plane nearest-neighbor 


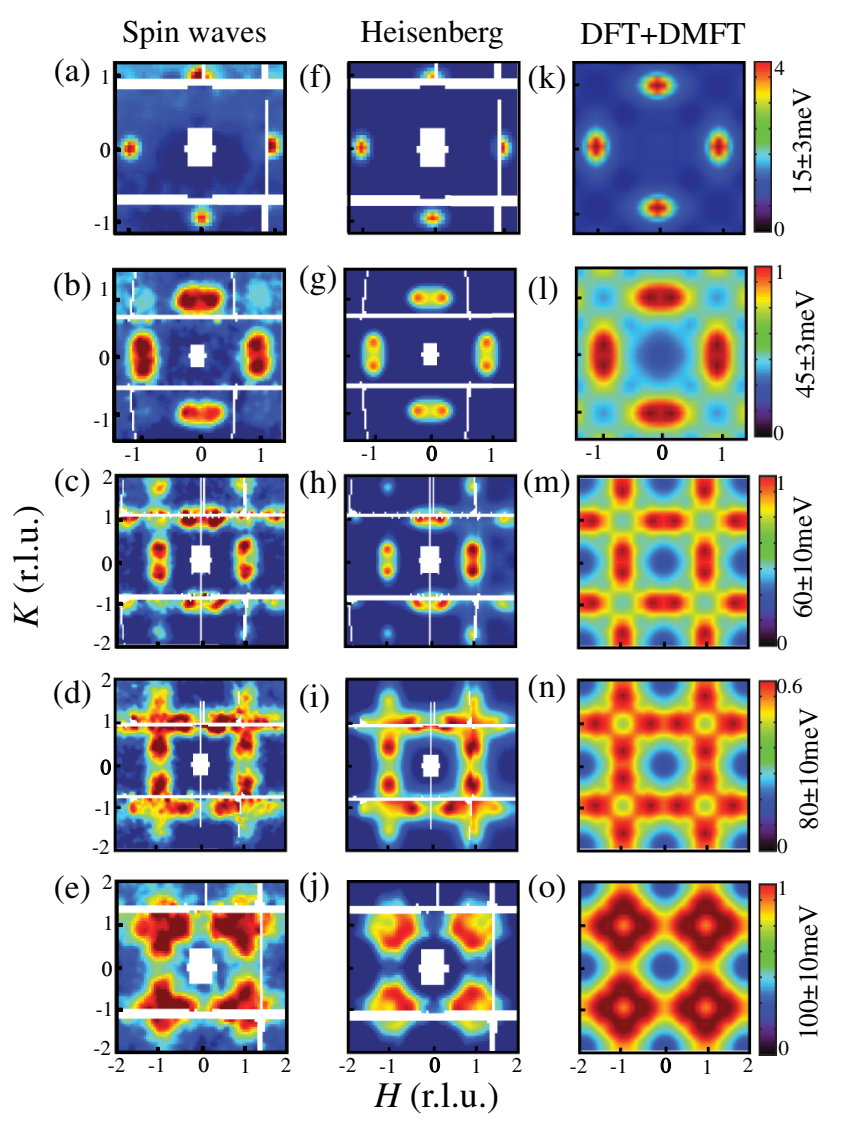

FIG. 2 (color online). Constant energy slices of the spin waves as a function of increasing energy at $5 \mathrm{~K}$ and fits using the Heisenberg Hamiltonian and DFT + DMFT method. Wave vector dependence of the spin waves for energy transfers of (a) $E=15 \pm 3 \mathrm{meV}\left[E_{i}=80 \mathrm{meV}\right.$ and $\left.\mathbf{q}=(H, K, 1.6)\right]$; (b) $E=$ $45 \pm 3 \mathrm{meV}\left[E_{i}=80 \mathrm{meV}\right.$ and $\left.\mathbf{q}=(H, K, 5)\right]$; (c) $E=60 \pm$ $10 \mathrm{meV} \quad\left[E_{i}=150 \mathrm{meV}\right.$ and $\left.\mathbf{q}=(H, K, 4.5)\right] ; \quad$ (d) $E=$ $80 \pm 10 \mathrm{meV}\left[E_{i}=150 \mathrm{meV}\right.$ and $\left.\mathbf{q}=(H, K, 6)\right]$; (e) $E=$ $100 \pm 10 \mathrm{meV}\left[E_{i}=250 \mathrm{meV}\right.$ and $\left.\mathbf{q}=(H, K, 5.5)\right]$. (f)-(j) Model calculation of identical slices by using the Heisenberg Hamiltonian with a new damping function convolved with the instrumental resolution [44]. (k)-(o) Calculations of identical energy slices from DFT + DMFT method. The color bars represent the vanadium-normalized absolute spin wave intensity in units of mbar/(sr MeV Fe atom).

[Fig. 1(a), $J_{1 a}$ and $J_{1 b}$ ], next-nearest-neighbor [Fig. 1(a), $\left.J_{2}\right]$, and out-of-plane $\left(J_{c}\right)$ exchange interactions with an isotropic spin-wave damping parameter $\Gamma$ suitable for the spin waves of $\mathrm{CaFe}_{2} \mathrm{As}_{2}$ [35]. Although allowing anisotropic spin wave damping parameter $\Gamma$ identical to that of $\mathrm{BaFe}_{2} \mathrm{As}_{2}$ [36] produces better fits, it still cannot describe the observed spin-wave spectra. Our best fits to the data were obtained by assuming a periodic array of two-dimensional Gaussian damping centers [44]. Figures 2(f)-2(j) show that the Heisenberg Hamiltonian with the effective magnetic exchange couplings $S J_{1 a}=40 \pm 0.8, S J_{1 b}=$ $16 \pm 0.6, S J_{2}=19 \pm 0.4$, and $S J_{c}=1.8 \pm 0.1 \mathrm{meV}$ fits the observed spin waves in Figs. 2(a)-2(e) quite well.

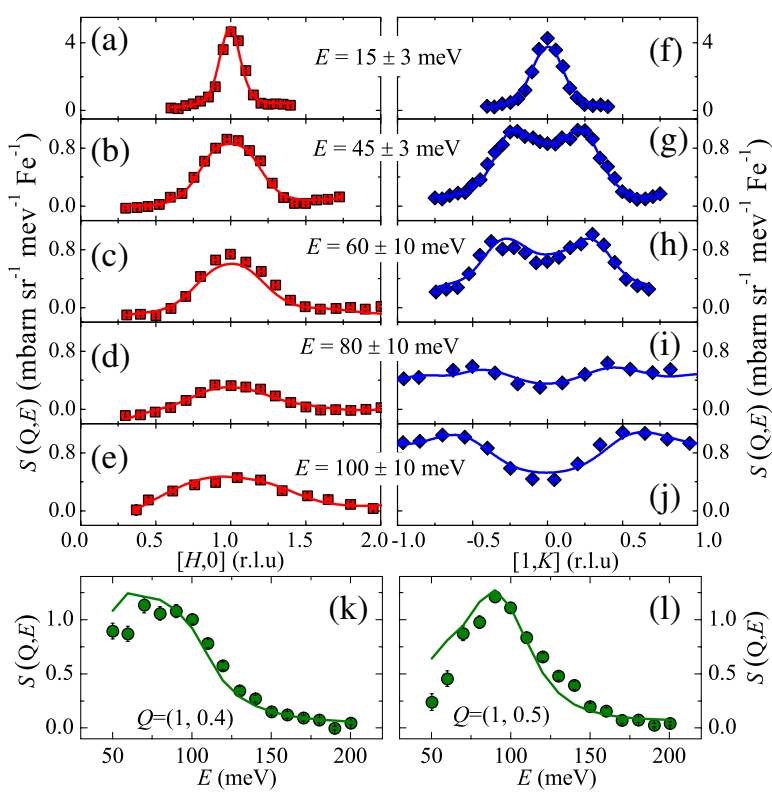

FIG. 3 (color online). Constant energy $(E)$ and wave vector(q) dependence of the spin wave excitations at $5 \mathrm{~K}$. Constant $E$ cuts at $E=15 \pm 3,45 \pm 3,60 \pm 10,80 \pm 10$, and $100 \pm 10 \mathrm{meV}$ along the $[H 0]$ direction (a)-(e) and along the $[1 K]$ direction (f)-(j). Constant $Q$ cuts at $Q=(1,0.4)$ (k) and $Q=(1,0.5)$ (l). The solid lines in (a)-(e), (f)-(j) and (k),(l) are global fits of our spin wave model discussed in the text.

Figures 2(k)-2(o) reveal wave vector dependence of spin waves calculated from a combined DFT + DMFT theory $[25,31,33]$. The spin excitations at different energies obtained from this approach have much similarities with the experimental data [Figs. 2(a)-2(e)]. The theoretical spin excitations are more diffusive than the experimental data primarily because the temperature used in the calculation $(116 \mathrm{~K})$ is higher $\left(T>T_{N}\right)$ than the temperature at which the experimental measurement was done $(5 \mathrm{~K})$.

To quantitatively compare the observed spin-wave spectra with fits from the Heisenberg Hamiltonian with anisotropic damping, we show in Figure 3 constant energy and constant wave vector cuts of spin waves and their comparison with the fits from the Heisenberg model. Figures 3(a)-3(e) show wave vector cuts along the $[H, 0]$ direction for spin-wave energies of $E=15 \pm 3,45 \pm 3$, $60 \pm 10,80 \pm 10$, and $100 \pm 10 \mathrm{meV}$. Figures $3(\mathrm{f})-3(\mathrm{j})$ plot cuts for these identical energies along the $[1, K]$ direction. Figures 3(k) and 3(l) are energy cuts at wave vectors $(1,0.4)$ and $(1,0.5)$, respectively. We see that the spin waves peak around $\sim 100 \mathrm{meV}$ near the zone boundary, much lower in energy than that of the $A \mathrm{Fe}_{2} \mathrm{As}_{2}$ [35-37].

Now we use the DFT + DMFT calculations to trace the origin of the peak position of the dynamical spin susceptibity $\chi^{\prime \prime}(q, E)$ at the zone boundary $[\mathbf{q}=(1,1, L)]$ and to understand the difference between $\mathrm{BaFe}_{2} \mathrm{As}_{2}$ and $\mathrm{NaFeAs}$. As shown in Fig. 4 , the $\chi^{\prime \prime}(q, E)$ at $\mathbf{q}_{\mathbf{M}}=(1,1,1)$ is peaked 


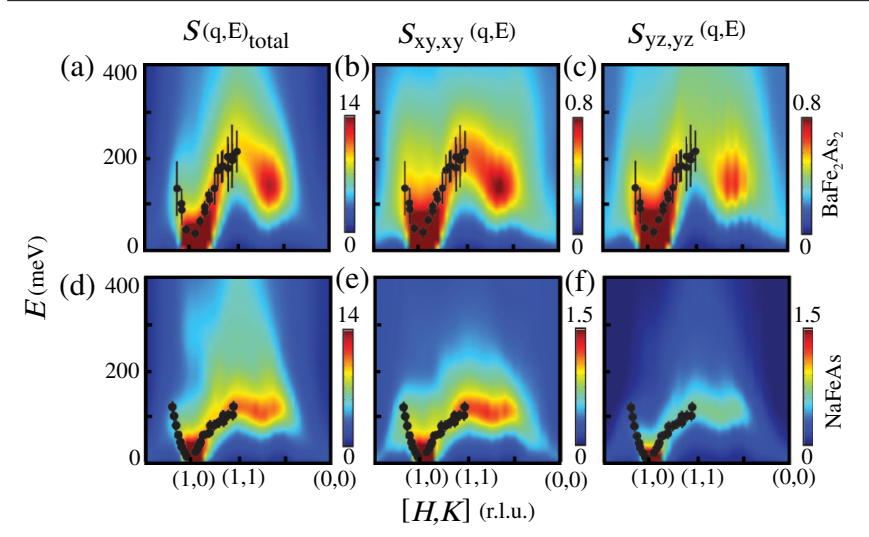

FIG. 4 (color online). Dynamic magnetic structure factor $S(q, E)$ for $\mathrm{BaFe}_{2} \mathrm{As}_{2}$ (top) and $\mathrm{NaFeAs}$ (bottom). The theoretical data are shown as colored plots while the black dots with verticalline error bars are experimental data. Panels (a) and (d) show the total $S(q, E)$, (b) and (e) the diagonal component $S_{x y, x y}(q, E)$ from the $d_{x y}$ orbital which has the largest contribution to the total $S(q, \omega)$ around the zone boundary $(1,1, L)$ points, and (c) and (f) the diagonal component $S_{y z, y z}(q, E)$ from the $d_{y z}$ orbital which contributes much less than the $d_{x y}$ orbital to the total $S(q, E)$ around the zone boundary $(1,1, \mathrm{~L})$ points. This orbital difference is enhanced in NaFeAs compared to $\mathrm{BaFe}_{2} \mathrm{As}_{2}$ due to the increased As height in $\mathrm{NaFeAs}$ and the kinetic frustration mechanism.

at $E \approx 0.2 \mathrm{eV}$ in $\mathrm{BaFe}_{2} \mathrm{As}_{2}$ and $E \approx 0.1 \mathrm{eV}$ in $\mathrm{NaFeAs}$. This peak is actually not associated with a peak in the imaginary (Im) part of the bare spin susceptibility $\operatorname{Im} \chi^{0}\left(q_{M}, E\right)$, i.e., it does not come from a particular nesting of the electronic structures. Actually, there is no peak structure in $\operatorname{Im} \chi^{0}\left(q_{M}, E\right)$ in the low-energy region as shown in Figs. S4(a) and S4(b) of the Supplemental Material [44]. This maximum of $\chi^{\prime \prime}\left(q_{M}, E\right)$ rather originates from the maximum of the real (Re) part of $\chi^{0}\left(q_{M}, E\right)$ as a result of the strong two-particle vertex interaction [45]. In both $\mathrm{BaFe}_{2} \mathrm{As}_{2}$ and $\mathrm{NaFeAs}$, the $d_{x y}$ orbital has the largest $\operatorname{Re} \chi^{0}(q, E)$ at low energy [see Figs. S4(a) and S4(b)], whose peak position is consistent with that in $\chi^{\prime \prime}(q, E)$ [Figs. 4(a) and 4(d), as well as Figs. S4(c) and $\mathrm{S} 4(\mathrm{~d})$ in [44]]. The peak position in $\chi^{\prime \prime}(q, E)$ at $q=$ $(1,1, L)$ is therefore associated with the peak position in the diagonal $d_{x y}$ component of $\operatorname{Re} \chi^{0}(q, E)$. Consequently, the much smaller bandwidth of the spin excitation in $\mathrm{NaFeAs}(E \approx 0.1 \mathrm{eV})$ compared to that of $\sim 0.2 \mathrm{eV}$ in $\mathrm{BaFe}_{2} \mathrm{As}_{2}$ can be explained by the fact that the electronic bandwidth of the $d_{x y}$ bands in NaFeAs is only about half the value in $\mathrm{BaFe}_{2} \mathrm{As}_{2}$ [44], due to the enhanced kinetic frustration mechanism induced by the increase of the As height [25]. Since the $d_{x y}$ orbital leads the contributions to the spin excitation near the zone boundary $\mathbf{q}=(1,1, L)$, and is more isotropic in the $x$ and $y$ directions, the increase of the As height combined with the kinetic frustration mechanism makes the effective nearest-neighbor Heisenberg exchange parameter more isotropic in NaFeAs than in $\mathrm{BaFe}_{2} \mathrm{As}_{2}$, as seen in the experiments.
From the energy dependence of the local dynamic spin susceptibility for $\mathrm{NaFeAs}$ and $\mathrm{BaFe}_{2} \mathrm{As}_{2}$ [Fig. 1(h)], we estimate that the total fluctuating magnetic moments for $\mathrm{NaFeAs}$ and $\mathrm{BaFe}_{2} \mathrm{As}_{2}$ are $\left\langle m^{2}\right\rangle \approx 3.2$ and $3.6 \mu_{B}^{2}$ per $\mathrm{Fe}$, respectively [46]. This means that the total fluctuating moments for both classes of materials are similar, corresponding to an effective spin $S=1 / 2$ per Fe $[28,32,46]$, in spite of their large differences in the static ordered moments and $T_{N}$ 's $[4,5]$. Therefore, while the increased iron pnictogen height in iron pnictides from $\mathrm{BaFe}_{2} \mathrm{As}_{2}$ to $\mathrm{NaFeAs}$ reduces the spin-wave bandwidth and increases the electron correlation effects, the total fluctuating moments remain essentially unchanged. Since the maximum $T_{c}$ of $\sim 22 \mathrm{~K}$ in Co-doped $\mathrm{NaFe}_{1-x} \mathrm{Co}_{x} \mathrm{As}$ is comparable to the $\sim 25 \mathrm{~K}$ for $\mathrm{BaFe}_{2-x} \mathrm{Co}_{x} \mathrm{As}_{2}$ [5], there does not appear to be a direct connection between the strength of electron correlations and high- $T_{c}$ superconductivity. For spin excitation mediated superconductors [13], high- $T_{c}$ superconductivity is controlled by the effective magnetic exchange coupling $J$ and the strength of electron-spin excitation coupling [32]. Since magnetic exchange couplings in $\mathrm{NaFeAs}$ are considerably smaller than those of the $\mathrm{BaFe}_{2} \mathrm{As}_{2}$, it would be interesting to determine if spin excitations are stronger in electron overdoped $\mathrm{NaFe}_{0.935} \mathrm{Co}_{0.045} \mathrm{As}$ [47] in comparison with the similar $T_{c} \mathrm{BaFe}_{1.9} \mathrm{Ni}_{0.1} \mathrm{As}_{2}$ [28].

In conclusion, our inelastic neutrons scattering experiments reveal that the effective magnetic exchange couplings in $\mathrm{NaFeAs}$ are smaller and more isotropic than those in the heavily studied $A \mathrm{Fe}_{2} \mathrm{As}_{2}$ family. These results are consistent with calculations from a DFT + DMFT method, suggesting that the increased pnictogen height from $A \mathrm{Fe}_{2} \mathrm{As}_{2}$ to $\mathrm{NaFeAs}$ plays a dominate role in increasing the electron correlations and fundamentally affects the spin dynamical properties in iron pnictides.

The single crystal growth and neutron scattering work at Rice, UTK was supported by the U.S. DOE, BES, through Contract No. DE-FG02-05ER46202 (P. D.). C. L.Z. and T. E. are partially supported by the U.S. DOE BES through the EPSCoR Grant No. DE-FG02-08ER46528. The DFT + DMFT computations were made possible by an Oak Ridge leadership computing facility director discretion allocation to Rutgers. The work at Rutgers is supported by DOE BES Grant No. DE-FG02-99ER45761 (Z.P. Y. and G. K.) and NSF-DMR 0746395 (K. H.). The work at Rice is also supported by NSF DMR-1308603 (P. D.). W.C.L is supported by NSF DMR-1104386. The work at SNS and HFIR was partially supported by the Division of Scientific User Facilities, U.S. DOE, BES. C. L. Zhang, L. W. Harriger, and Z. P. Yin made equal contributions to this work.

pdai@rice.edu

[1] Y. Kamihara, T. Watanabe, M. Hirano, and H. Hosono, J. Am. Chem. Soc. 130, 3296 (2008).

[2] C. de la Cruz et al., Nature (London) 453, 899 (2008). 
[3] C. W. Chu, F. Chen, M. Gooch, A. M. Guloy, B. Lorenz, B. Lv, K. Sasmal, Z. J. Tang, J. H. Tapp, and Y. Y. Xue, Physica (Amsterdam) 469C, 326 (2009).

[4] S. L. Li, C. de la Cruz, Q. Huang, G. Chen, T.-L. Xia, J. Luo, N. Wang, and P. C. Dai, Phys. Rev. B 80, 020504(R) (2009).

[5] D. C. Johnston, Adv. Phys. 59, 803 (2010).

[6] P. C. Dai, J. P. Hu, and E. Dagotto, Nat. Phys. 8, 709 (2012).

[7] I. I. Mazin, D. J. Singh, M. D. Johannes, and M. H. Du, Phys. Rev. Lett. 101, 057003 (2008).

[8] D. J. Singh and M.-H. Du, Phys. Rev. Lett. 100, 237003 (2008).

[9] K. Haule, J. H. Shim, and G. Kotliar, Phys. Rev. Lett. 100, 226402 (2008).

[10] Q. Si and E. Abrahams, Phys. Rev. Lett. 101, 076401 (2008).

[11] C. Fang, H. Yao, W. F. Tsai, J. P. Hu, and S. A. Kivelson, Phys. Rev. B 77, 224509 (2008).

[12] C. K. Xu, M. Müller, and S. Sachdev, Phys. Rev. B 78, 020501(R) (2008)

[13] D. J. Scalapino, Rev. Mod. Phys. 84, 1383 (2012).

[14] E. Fawcett, Rev. Mod. Phys. 60, 209 (1988).

[15] P. J. Hirschfeld, M. M. Korshunov, and I. I. Mazin, Rep. Prog. Phys. 74, 124508 (2011).

[16] A. Chubukov, Annu. Rev. Condens. Matter Phys. 3, 57 (2012).

[17] Z. P. Yin, S. Lebégue, M. J. Han, B. P. Neal, S. Y. Savrasov, and W. E. Pickett, Phys. Rev. Lett. 101, 047001 (2008).

[18] C. C. Lee, W. G. Yin, and W. Ku, Phys. Rev. Lett. 103, 267001 (2009).

[19] F. Krüger, S. Kumar, J. Zaanen, and J. van den Brink, Phys. Rev. B 79, 054504 (2009).

[20] W. C. Lv, J. S. Wu, and P. Phillips, Phys. Rev. B 80, 224506 (2009).

[21] C. C. Chen, J. Maciejko, A. P. Sorini, B. Moritz, R. R. P. Singh, and T. P. Devereaux, Phys. Rev. B 82, 100504(R) (2010).

[22] M. M. Qazilbash, J. J. Hamlin, R. E. Baumbach, L. Zhang, D. J. Singh, M. B. Maple, and D. N. Basov, Nat. Phys. 5, 647 (2009).

[23] D. N. Basov and A. V. Chubukov, Nat. Phys. 7, 272 (2011).

[24] K. Haule and G. Kotliar, New J. Phys. 11, 025021 (2009).

[25] Z. P. Yin, K. Haule, and G. Kotliar, Nat. Mater. 10, 932 (2011).

[26] P. A. Lee, N. Nagaosa, and X.-G. Wen, Rev. Mod. Phys. 78, 17 (2006).

[27] Z. P. Yin, K. Haule, and G. Kotliar, Nat. Phys. 7, 294 (2011)

[28] M. S. Liu et al., Nat. Phys. 8, 376 (2012).
[29] G. Kotliar, S. Y. Savrasov, K. Haule, V. S. Oudovenko, O. Parcollet, and C. A. Marianetti, Rev. Mod. Phys. 78, 865 (2006).

[30] K. Haule, C.-H. Yee, and K. Kim, Phys. Rev. B 81, 195107 (2010).

[31] H. Park, K. Haule, and G. Kotliar, Phys. Rev. Lett. 107, 137007 (2011).

[32] M. Wang et al., Nat. Commun. 4, 2874 (2013).

[33] Z. P. Yin, K. Haule, and G. Kotliar, arXiv:1311.1188.

[34] S. O. Diallo et al., Phys. Rev. Lett. 102, 187206 (2009).

[35] J. Zhao, D. T. Adroja, D.-X. Yao, R. Bewley, S. Li, X. F. Wang, G. Wu, X. H. Chen, J. Hu, and P. C. Dai, Nat. Phys. 5, 555 (2009).

[36] L. W. Harriger, H. Q. Luo, M. S. Liu, C. Frost, J. P. Hu, M. R. Norman, and P. C. Dai, Phys. Rev. B 84, 054544 (2011).

[37] R. A. Ewings, T. G. Perring, J. Gillett, S. D. Das, S. E. Sebastian, A. E. Taylor, T. Guidi, and A. T. Boothroyd, Phys. Rev. B 83, 214519 (2011).

[38] J. T. Park et al., Phys. Rev. B 86, 024437 (2012).

[39] D. R. Parker, M. J. P. Smith, T. Lancaster, A. J. Steele, I. Franke, P. J. Baker, F. L. Pratt, M. J. Pitcher, S. J. Blundell, and S. J. Clarke, Phys. Rev. Lett. 104, 057007 (2010).

[40] M. A. Tanatar, N. Spyrison, K. Cho, E. C. Blomberg, G. Tan, P. C. Dai, C. Zhang, and R. Prozorov, Phys. Rev. B 85, 014510 (2012).

[41] G. T. Tan et al., Phys. Rev. B 87, 144512 (2013).

[42] Y. Song, S. V. Carr, X. Lu, C. Zhang, Z. C. Sims, N. F. Luttrell, S. Chi, Y. Zhao, J. W. Lynn, and P. C. Dai, Phys. Rev. B 87, 184511 (2013).

[43] C. Lester, J.-H. Chu, J. G. Analytis, T. G. Perring, I. R. Fisher, and S. M. Hayden, Phys. Rev. B 81, 064505 (2010).

[44] See Supplemental Material http://link.aps.org/supplemental/ 10.1103/PhysRevLett.112.217202 for more details.

[45] Since the $d_{x y}$ orbital has the largest contribution [Figs. S4(a)-S4(b)] and for illustration purpose, we consider the single-orbital case, in which the complex two-particle irreducible vertex function is replaced by a single number $U$. Therefore, we have $\chi^{\prime \prime}(q, E)=\frac{\operatorname{Im} \chi^{0}(q, E)}{\left(1-U \operatorname{Re} \chi^{0}(q, E)\right)^{2}+\left(U \operatorname{Im} \chi^{0}(q, E)\right)^{2}}$. Since $\left(U \operatorname{Im} \chi^{0}\right)^{2}$ is very small at small $E$ [Figs. S4(a) and $\mathrm{S} 4(\mathrm{~b})]$, the peak position in $\chi^{\prime \prime}(q, E)$ is mainly determined by $U \operatorname{Re} \chi^{0}(q, E)$ approaching unity. Hence, the peak position in $\chi^{\prime \prime}(q, E)$ is connected to the peak position in $\operatorname{Re} \chi^{0}(q, E)$.

[46] L. W. Harriger, M. Liu, H. Luo, R. A. Ewings, C. D. Frost, T. G. Perring, and P. C.Dai, Phys. Rev. B 86, 140403(R) (2012).

[47] C. L. Zhang et al., Phys. Rev. B 88, 064504 (2013). 